\title{
Concurrent Bilateral Emphysematous Pyelonephritis and Emphysematous Cystitis: A Case Report of Glomerulonephritis Severe Complication
}

\author{
Michał Godzisz (D) \\ Przemysław Mitura (ID \\ Damian Widz (iD \\ Damian Sudot (iD) \\ Iga Kuliniec \\ Krzysztof Bar (D)
}

Department of Urology and Urologic Oncology, Medical University of Lublin, Lublin, Poland
Correspondence: Michał Godzisz Medical University of Lublin, Jaczewskiego 8, 20-954 Lublin, 20-819, Poland Tel +48660346267

Email Michal.godzisz92@gmail.com

\begin{abstract}
Emphysematous pyelonephritis (EPN) and emphysematous cystitis (EC) are severe, necrotizing emphysematous urinary tract infections (EUTI) characterized by the presence of gas in the lumen and wall of the urinary tract and renal parenchyma. EUTIs are quite uncommon, but combination of bilateral EPN and EC is extremely rare. We report a case of 57-year-old diabetic female diagnosed with septic shock due to concurrent bilateral EPN and EC. Patient was successfully treated with a drainage of pelvicalyceal systems and the bladder combined with a conservative approach. This unique case presents a possible association between glomerulonephritis and EUTI.
\end{abstract}

Keywords: emphysematous pyelonephritis, emphysematous cystitis, glomerulonephritis, diabetes, septic shock, urinary tract infections

\section{Introduction}

Emphysematous pyelonephritis (EPN) and emphysematous cystitis (EC) are severe, necrotizing emphysematous urinary tract infections (EUTI) characterized by the presence of gas in the lumen and wall of the urinary tract, in renal parenchyma, or near retroperitoneal tissue. The known risk factors for EUTI are advanced age (6070 years), diabetes, female gender, immunosuppression, recurrent urinary tract infections, neurogenic bladder, indwelling catheter, and bladder outlet obstruction, alcohol. ${ }^{1,2}$ These diseases may have varied presentations ranging from asymptomatic infection, dysuria, pneumaturia, fever, and abdominal pain to septic shock. Concurrent EUTIs are noted in $15.4 \%$ of all the cases of EC. ${ }^{3}$ The death rate of combined EUTIs is $14 \% .{ }^{1}$ However, the literature reports a mortality rate of $54 \%$ in patients with secondary shock to EPN. ${ }^{4}$ The main condition associated with the development of EUTI is the presence of gas-forming bacteria, of which the most frequently detected is Escherichia coli. The most recent and largest pooled EUTI analysis so far included 106 articles, 136 case reports, in which only 21 patients had had EC associated with EUTI other than EC, most frequently EPN. ${ }^{3}$ The aim of this EUTI case report is to make a significant contribution to the knowledge of its etiology, course, diagnostic methods, and treatment options.

\section{Case Report}

A 57-year-old female patient was admitted to the emergency department with a 2-day history of hyperglycemia ( $>500 \mathrm{mg} \%$ ), diffused abdominal pain dominant 
in the suprapubic region, and fever. The patient had a history of multiple comorbidities, which included steroid treatment-related diabetes and osteoporosis, chronic renal disease, submicroscopic glomerulonephritis with progression to focal segmental glomerulosclerosis, ischemic heart disease, gout, carotid atherosclerosis, and obesity. Her vital signs were in the normal range: saturation $94-95 \%$, blood pressure 130/80 $\mathrm{mmHg}$, heart rate $90 / \mathrm{min}$, respiration rate 16/min, Glasgow Coma Scale 15, and body temperature of $36.6^{\circ} \mathrm{C}$. The only deviation in the physical examination was generalized tenderness of the abdominal cavity, which was dominant in the inferior part. No abdominal guarding or rebound tenderness was observed.

Due to the extremely unfavorable laboratory test results at admission (Table 1) and rapid worsening of the patient's general condition, an urgent computed tomography (CT) scan of the chest, abdomen, and pelvis was performed. It revealed the presence of gas bubbles in the lumen of the bladder, the bladder wall, and in both pelvicalyceal systems. (Figures 1 and 2) The diagnosis of bilateral EPN and EC was established, and the patient was admitted to the Department of Urology. She was started immediately on empirical broad-spectrum antibiotic therapy. The Single-J (SJ) 8Fr catheters were urgently inserted into both the kidneys and an 18Fr Foley catheter into the bladder, resulting in an outflow of purulent urine, which was collected for culture. Due to septic shock and acute renal failure, high doses of vasopressors were administered and hemodialysis was performed twice. Due to the treatment, the patient's general condition improved, inflammation decreased, kidney function improved, and acid-base and electrolyte balance normalized, and so the doses of vasopressors were reduced. Urine culture revealed $E$. coli susceptible to all the antibiotics used-

Table I Patient Laboratory Test Results at Admission to the Hospital

\begin{tabular}{|l|l|}
\hline Test & Result \\
\hline White blood count & $85.72 \times 10^{\wedge} 9 / \mathrm{L}$ \\
Hemoglobin & $9.9 \mathrm{~g} / \mathrm{dL}$ \\
C-reactive protein & $392 \mathrm{mg} / \mathrm{dL}$ \\
Procalcitonin & $1000 \mathrm{ng} / \mathrm{mL}$ \\
Serum creatinine & $5.1 \mathrm{mg} / \mathrm{dL}$ \\
Serum urea & $327.2 \mathrm{mg} / \mathrm{dL}$ \\
Serum potassium ion & $7.4 \mathrm{mmol} / \mathrm{L}$ \\
Serum sodium ion & $133 \mathrm{mmol} / \mathrm{L}$ \\
INR & 1.5 \\
Urinalysis & Bacteriuria and pyuria \\
\hline
\end{tabular}

antibiotic therapy was continued. On the 4th day of hospitalization, the patient was transferred to the nephrology ward for further treatment. Vasopressors were discontinued on the 14th day of hospitalization. Control CT scan of the abdomen and pelvis was performed on the 27 th day which revealed complete regression of the imaging findings of the disease. (Figures 3 and 4) The SJs and Foley catheters were removed on the following day. The patient was discharged in good general condition on the 29 th day.

\section{Discussion}

The patient presented in this case report had several risk factors of EUTI: female sex, age, diabetes, and immunosuppression (chronic treatment with high dosage of steroids). The literature shows that women are two times more susceptible to EUTI and $60-70 \%$ of EUTI patients had diabetes. ${ }^{1,3}$ The patient in this case report suffered from nephrotic syndrome in the course of microscopic glomerulonephritis. Albumins resulting from proteinuria by microscopic glomerulonephritis can be the substrates for gas-forming bacteria. ${ }^{5}$ The mortality of ETUI has been reported to range around 7-25\% depending on the coexistence of EC and EPN., 6

The symptoms of EPN and EC are similar to those of ordinary cystitis and pyelonephritis, and include dysuria, fever/rigors, nausea, vomiting, abdominal pain, and flank pain, but most often the disease takes a much more severe course. EUTI may be asymptomatic or cause a sudden septic shock, which was the case in our patient.

The most common pathogens detected in urine and blood are E. coli and the second most common is Klebsiella pneumonia. $^{8}$ Other pathogens include Pseudomonas aeruginosa, Proteus mirabilis, Candida albicans and C. tropicalis, Aspergillus fumigatus, Staphylococcus aureus, Group D Streptococcus, Enterococcus faecalis, Enterobacter aerogenes, and Clostridium perfringens, and $\mathrm{Cl}$. welchii.

The diagnosis of EUTI is most commonly based on imaging. A plain radiograph shows an abnormal gas shadow in the renal bed or/and curvilinear area of radiolucency delineating the bladder wall with or without intraluminal air. Ultrasonography and CT of the abdomen and pelvis can help in identifying the presence of gas in the bladder and renal parenchyma. CT is recommended because it is the most accurate technique and provides additional information needed for differential diagnosis and planned surgical treatment.

Conservative treatments and drainage of the urinary tract and fluid reservoirs in the retroperitoneal space are 


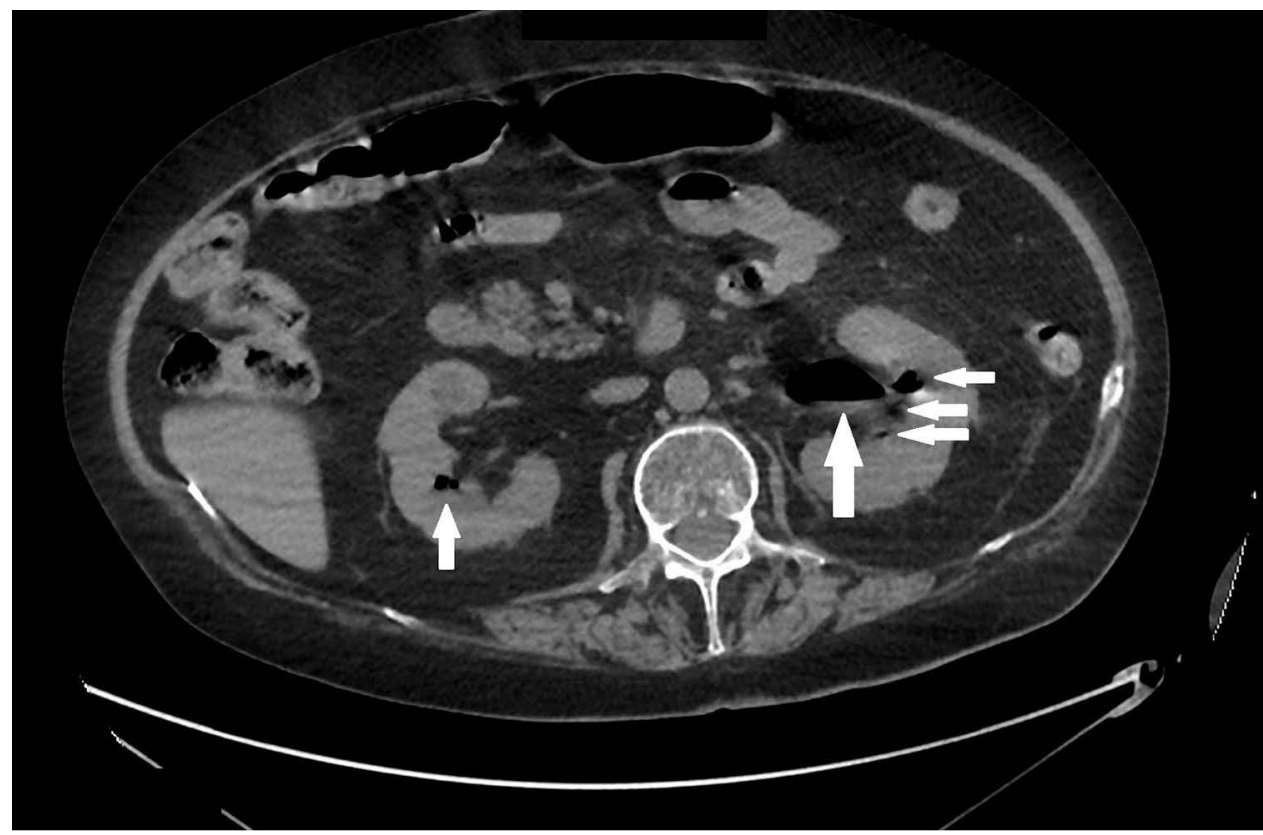

Figure I The coronal non-contrast-enhanced computed tomography (CT) scan showing the presence of gas in pelvicalyceal systems of both kidneys (white arrows). CT scans were taken at admission to the hospital.

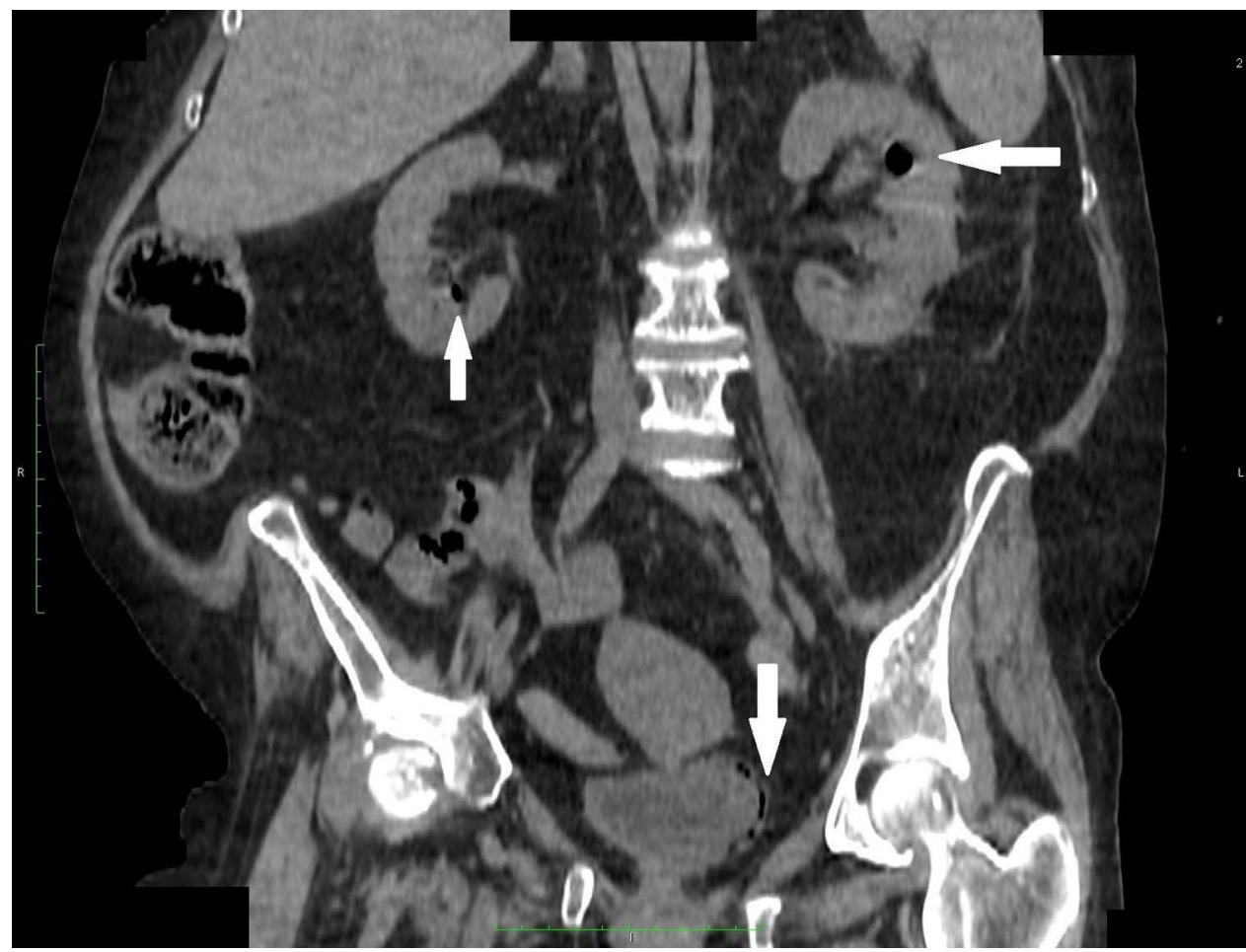

Figure 2 The axial non-contrast-enhanced computed tomography scan showing the presence of gas in pelvicalyceal systems of both kidneys (white arrows) and gas foci in the bladder wall. CT scans were taken at admission to the hospital.

the basis of the treatment of EPN and EC and are associated with the lowest mortality (13.5\%). These treatments include broad-spectrum antibiotic therapy, oxygen therapy, fluid and acid-base balance, use of vasopressors in the case of septic shock, and initiation of hemodialysis in the case of acute renal failure. Antibiotic therapy and percutaneous drainage allowed maintaining the function of the affected kidney in $70 \%$ 


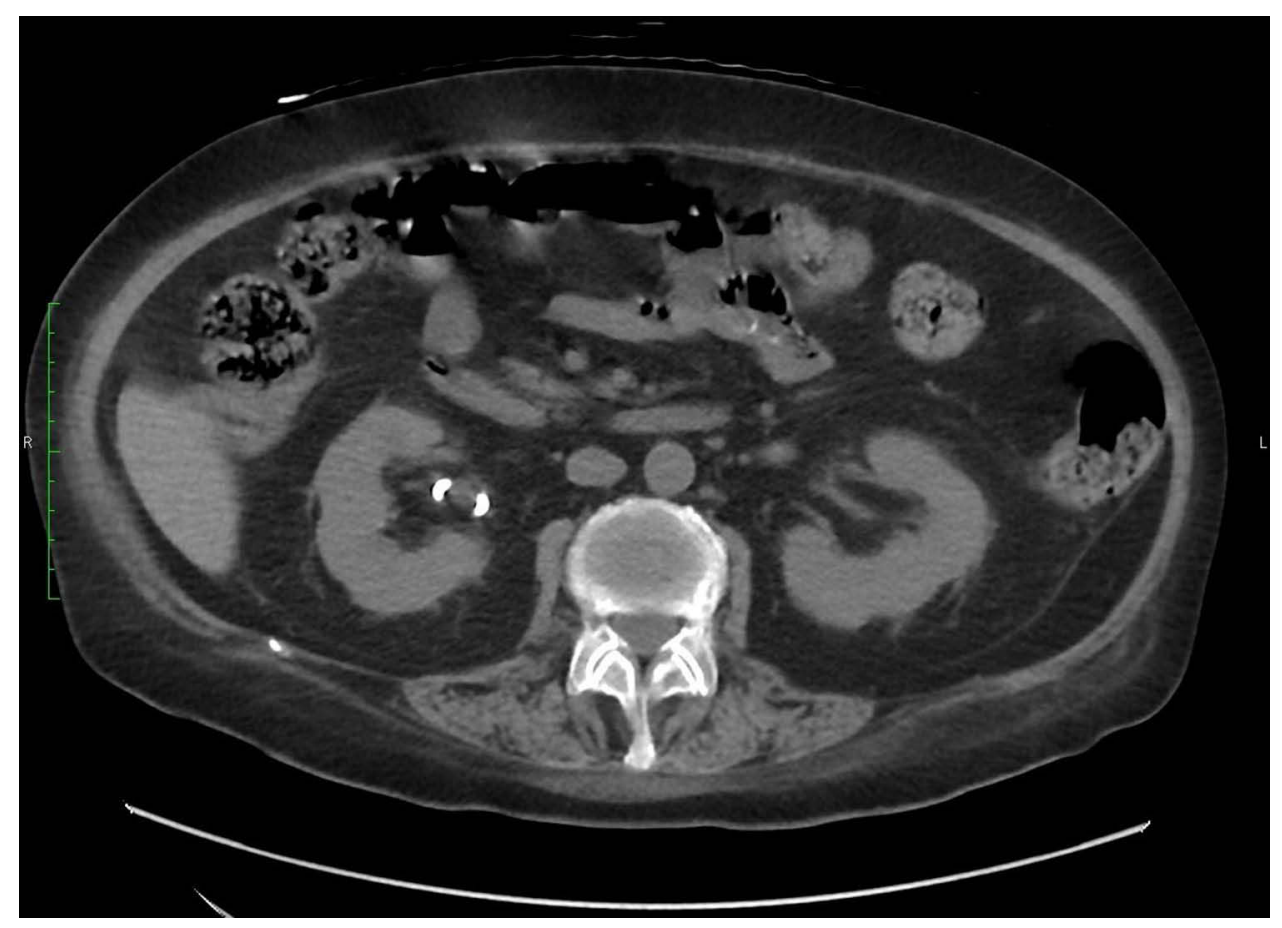

Figure 3 The coronal non-contrast-enhanced computed tomography (CT) scan (The kidneys level) revealed complete regression of the imaging findings of the disease. CT scans were taken on the 27 th day of hospitalization.

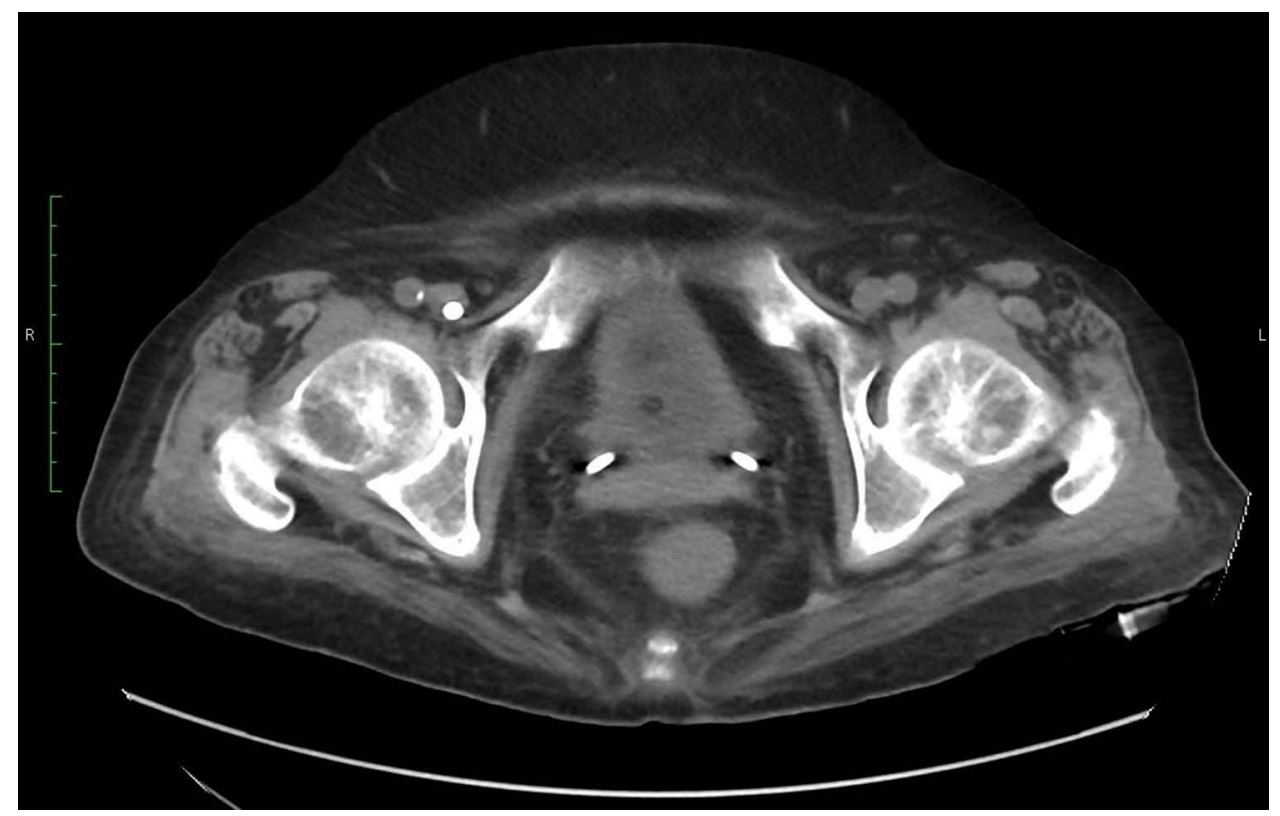

Figure 4 The coronal non-contrast-enhanced computed tomography (CT) scan (The bladder level) revealed complete regression of the imaging findings of the disease. CT scans were taken on the 27 th day of hospitalization.

of the cases. Drainage should be performed until the radiological features of EUTI have completely receded. ${ }^{6}$ In the nonresponder group, surgical treatment is indicated, which includes the removal of infected, necrotic tissues and drainage of the retroperitoneal and perivesical space in the classic way. If debridement is not sufficient, partial cystectomy, or cystectomy and/or nephrectomy might be required. ${ }^{9}$ According to the metaanalyses by Somani et al, surgical management was essential in $13 \%$ of the patients. ${ }^{6}$ 


\section{Conclusion}

Despite their severe course and serious prognosis, EC and EPN can be effectively treated. EUTI is a group of rare disorders, which makes it difficult to conduct a prospective study with high-quality evidence. The publications in the form of EUTI clinical cases collected over decades have contributed to increasing the effectiveness of diagnostic and therapeutic management strategies. Furthermore, they have allowed formulating hypotheses about the etiology of this disease. Thus, each described case of this rare disease adds value to evidence-based medicine and improves patients' prognosis. We believe that, in this case, EUTI was caused by the chain of events related to glomerulonephritis. Glomerulonephritis caused nephrotic syndrome, which was treated with steroids. Steroids induced diabetes, which with other risk factors contributed to EUTI.

\section{Abbreviations}

EPN, emphysematous pyelonephritis; EC, emphysematous cystitis; EUTI, emphysematous urinary tract infections; CT, computed tomography; SJ, Single-J.

\section{Ethics Approval and Informed Consent}

Written informed consent has been provided by the patient to have the case details and any accompanying images published. Institutional approval was not required to publish the case details.

\section{Author Contributions}

All authors made a significant contributions to the idea and design, acquisition of data, analysis and interpretation of data; took part in drafting the article or revising it critically for important intellectual content; agreed to submit the article to the Research and Reports in Urology; approved the version to be published; agree to be accountable for all aspects of the work.

\section{Disclosure}

The authors report no conflicts of interest in this work.

\section{References}

1. Thomas AA, Lane BR, Thomas AZ, Remer EM, Campbell SC, Shoskes DA. Emphysematous cystitis: a review of 135 cases. BJU Int. 2007;100(1):17-20. doi:10.1111/j.1464-410X.2007.06930.x

2. Sanc1 A, Aydoğ E, Karaburun MC, Süer E. A case of alcohol-related emphysematous cystitis. Ukr J Nephrol Dial. 2019;1(1(65)):3-6. doi:10.31450/ukrjnd.1(65).2020.01

3. Schicho A, Stroszczynski C, Wiggermann P. Emphysematous cystitis: mortality, risk factors, and pathogens of a rare disease. Clin Pract. 2017;7(2). doi:10.4081/cp.2017.930

4. Ubee SS, McGlynn L, Fordham M. Emphysematous pyelonephritis. BJU Int. 2011;107(9):1474-1478. doi:10.1111/j.1464-410X.20 10.09660.x

5. Hawtrey CE, Williams JJ, Schmidt JD. Cystitis emphysematosa. Urology. 1974;3(5):612-614. doi:10.1016/S0090-4295(74)80259-1

6. Somani BK, Nabi G, Thorpe P, Hussey J, Cook J, N'Dow J. Is percutaneous drainage the new gold standard in the management of emphysematous pyelonephritis? Evidence from a systematic review. J Urol. 2008;179(5):1844-1849. doi:10.1016/j.juro.2008.01.019

7. Falagas ME, Alexiou VG, Giannopoulou KP, Siempos II. Risk factors for mortality in patients with emphysematous pyelonephritis: a meta-analysis. $J$ Urol. 2007;178(3):880-885. doi:10.1016/j. juro.2007.05.017

8. Huang JJ, Tseng CC. Emphysematous pyelonephritis: clinicoradiological classification, management, prognosis, and pathogenesis. Arch Intern Med. 2002;160(6):797-805. doi:10.1001/archinte.160.6.797

9. Hudnall MT, Jordan BJ, Horowitz J, Kielb S. A case of emphysematous cystitis and bladder rupture. Urol Case Rep. 2019;24.

\section{Publish your work in this journal}

Research and Reports in Urology is an international, peer-reviewed, open access journal publishing original research, reports, editorials, reviews and commentaries on all aspects of adult and pediatric urology in the clinic and laboratory including the following topics: Pathology, pathophysiology of urological disease; Investigation and treatment of urological disease; Pharmacology of drugs used for the treatment of urological disease. The manuscript management system is completely online and includes a very quick and fair peer-review system, which is all easy to use. Visit http://www.dovepress.com/ testimonials.php to read real quotes from published authors. 